\title{
Presuppositions ANd Local ConteXts ${ }^{1}$
}

\author{
PHILIPPE SCHLENKER \\ Institut Jean-Nicod and New York University
}

\begin{abstract}
In the last thirty years, the problem of presupposition projection has been taken to provide a decisive argument for a dynamic approach to meaning, one in which expressions are not evaluated with respect to the 'global' context of utterance, but rather with respect to a 'local context' obtained by updating the global one with expressions that occur earlier in the sentence. The computation of local contexts is taken by dynamic analyses to follow from a generalization of the notion of belief update. We argue that the dynamic approach is faced with a dilemma: in its pragmatic incarnation (Stalnaker), it is explanatory but not general; in its semantic incarnation (Karttunen and Heim), it is general but not explanatory. We suggest that the dilemma stems for a faulty understanding of 'local contexts', and we offer a new reconstruction of this notion which eschews belief update but offers a general and fully precise solution to the projection problem.
\end{abstract}

Keywords: presupposition, dynamic semantics, trivalence, presupposition projection, semantics, pragmatics

For the last thirty years, it has been assumed that a presupposition must be entailed (or 'satisfied') by the context, construed as what is common belief between the speaker and addressee: John has stopped smoking is acceptable in a context $\mathrm{C}$ just in case $\mathrm{C}$ entails that John used to smoke. The context in this sense is often called the 'context set', identified for simplicity with the set of possible worlds compatible with what the speech act participants take for granted (Stalnaker 1978, 2002); in this case, then, the requirement is that each world in C is one in which John used to smoke. But to explain how the presuppositions of complex sentences are computed, this analysis must be supplemented with a dynamic view of context change. This is because presuppositions are sometimes filtered out in complex sentences. For instance, the conjunction John used to smoke and he has stopped asserts rather than presupposes that John smoked. The standard explanation (Stalnaker 1974; Karttunen 1974; Heim 1983) is that the second conjunct is not evaluated with respect to the initial context, but rather with respect to a 'local context', obtained by updating the initial one with the content of the first conjunct.

\footnotetext{
${ }^{1}$ A technical and much longer presentation of this theory is offered in Schlenker, to appear. Many thanks to Emmanuel Chemla, Paul Egré, Dan Lassiter and Benjamin Spector for helpful remarks related to this paper.
} 
Depending on the approach, context change is effected through semantic or pragmatic rules. In either case, a clause $H$ evaluated in a context $\mathrm{C}$ gives rise to a new context, written as $\mathrm{C}[\mathrm{H}]$ ('the update of $\mathrm{C}$ with the formula $H$ '). If $H$ is an elementary clause $p$ which has no presupposition, $\mathrm{C}[\mathrm{H}]$ is always defined, and it is just the set of $\mathrm{C}$-worlds that satisfy $p$. If $H$ is an elementary clause with a presupposition $p$ and an assertive component $p$ ' (which we write as $p p^{\prime}$, with the convention that presuppositions are underlined), $\mathrm{C}[\mathrm{H}]$ is undefined in case some world in $\mathrm{C}$ fails to satisfy the presupposition $p$; if it is defined, $\mathrm{C}[\mathrm{H}]$ is the set of $\mathrm{C}$-worlds that satisfy the assertive component $p$ '. These rules are summarized in (1), where undefinedness is written as \#:

(1) a. $C[p]=\left\{w \in C: p^{\prime}\right.$ is true in $\left.w\right\}$

b. $C\left[p p^{\prime}\right]=\#$ iff for some world $w$ in $C, p$ is false in $w$. If $C\left[p p^{\prime}\right] \neq \#, C[p p ']=\{w \in C$ : p' is true in $w$ \}

Context update is then extended to conjunctions by postulating that the update of a context $\mathrm{C}$ with $F$ and $G$ is the successive update of $\mathrm{C}$ with $F$, and then with $G$, as is defined in (2)a and illustrated in (2)b.

(2) a. $\mathrm{C}[\mathrm{F}$ and $\mathrm{G}]=(\mathrm{C}[\mathrm{F}])[\mathrm{G}]$

b. $\mathrm{C}[$ John used to smoke and he has stopped smoking $]=(\mathrm{C}[$ John used to smoke $])[$ he has stopped smoking]

In our example, John used to smoke is non-presuppositional, and thus C[John used to smoke] is the set of C-worlds in which John smoked - which means that the presupposition of the second conjunct (he has stopped smoking) is automatically satisfied in its local context.

This analysis has proven extremely influential, and it has convinced many linguists and philosophers that semantics and pragmatics should be studied within a dynamic framework. But the dynamic approach has been caught in a theoretical dilemma: in its pragmatic incarnation, it is explanatory but insufficiently general; in its semantic version, it is general but it lacks explanatory force. We will argue that the source of the dilemma lies in a faulty understanding of 'local contexts', and that the problem can be solved once this notion is reconstructed in a different way.

\section{The Dilemma}

\subsection{The Pragmatic Horn}

Following Stalnaker's work, some have taken context update to result from a rational process of information exchange: as soon as the addressee hears John used to smoke, and unless he objects, he changes his beliefs by accepting this proposition. This was taken to justify the update rule in (2). This approach has great initial force because the assertion of a conjunction can plausibly be equated with the successive assertion of each conjunct. But it doesn't easily extend to other complex sentences, such as those in (3).

(3) a. It didn't rain or it has stopped raining (Presupposition: none)

b. Each of my students has stopped smoking (Presupposition: each of my students used to smoke) 
c. None of my students have stopped smoking (Presupposition: each of my students used to smoke)

The point of a disjunction is precisely that one can assert it without being committed to either disjunct; this makes it difficult to see how an assertion-based analysis can be applied to (3)a, despite the fact that there are non-trivial presuppositional facts to account for. In this case, it is the negation of the first disjunct that serves to justify the presupposition of the second one; this fact should presumably follow from a principled account of presupposition projection. When the presuppositional expression is predicative rather than propositional, as is the case in (3)b-c, things are equally difficult: the complex predicate has stopped smoking interacts with the quantifier so as to yield a presupposition that each of my students used to smoke (see Chemla, to appear, for experimental results that confirm this fact with a variety of presupposition triggers). So the local context of has stopped smoking must entail the presupposition used to smoke. This requirement can easily be formalized with a generalized notion of entailment (among predicates), but the difficulty is that a predicative element just isn't the right kind of object to apply belief update to. More generally, it is unclear how Stalnaker's pragmatic analysis can be applied at the sub-propositional level, as seems to be required here. No detailed account of these cases has been offered along Stalnakerian lines - and the difficulties to be overcome appear to be non-trivial.

\subsection{The Semantic Horn}

Since Stalnaker's pragmatic rationale appeared difficult to extend, Heim 1983 (following in part Karttunen 1974) gave a semantic version of the dynamic analysis, one in which the very meaning of words is dynamic from the start. The update rule in (2) is thus preserved, but it is taken to follow from the lexical meaning of and rather than from a procedure of belief update. The meaning of each word is thus re-analyzed in terms of context change potentials, which are functions from contexts to contexts. It was initially thought that context change potentials could be predicted from truth-conditional properties alone, a claim that Heim later retracted (Heim 1992 fn. 9). For as was noted in the 1980s (e.g. Soames 1989), dynamic semantics is so powerful that it can stipulate in the lexical entry of any operator the way in which it transmits presuppositions. For this reason, the framework is insufficiently explanatory: any classical operator can be given a variety of dynamic meanings which agree on non-presuppositional sentences, but make conflicting predictions about presuppositional ones; which shows that the dynamic framework fails to predict presupposition projection from truth-conditional content.

To make the point concrete, we can observe that dynamic semantics can define a 'deviant' conjunction and* as in (4):

$$
\mathrm{C}[\mathrm{F} \text { and } * \mathrm{G}]=(\mathrm{C}[\mathrm{G}])[\mathrm{F}]
$$

In non-presuppositional examples, and* has the same effect as and: it returns the set of C-worlds that satisfy both $F$ and $G$. But in examples such as John used to smoke and he has stopped, (4) predicts that the sentence should result in a presupposition failure because $\mathrm{C}$ is first updated with the second conjunct before it is updated with the first one. The difficulty is that dynamic semantics has no independent way of ruling out connectives such as and*; taken literally, the framework predicts that such a connective could exist in the world's languages, which does not appear to be correct. Furthermore, the problem can be reproduced with all other connectives and operators: dynamic semantics is so powerful that it lacks explanatory force. 


\section{A New Solution}

We claim that the dilemma stems from a faulty understanding of local contexts. Local contexts play a role in a variety of sub-propositional environments, which means that an account in terms of belief update is probably ill-suited to the task: it must forego generality (the pragmatic horn), or abandon its pragmatic inspiration to be general, but stipulative (the semantic horn). By offering a new analysis of local contexts, we will develop an account that retains the pragmatic inspiration of Stalnaker's analysis while preserving the formal precision and generality of dynamic semantics.

\subsection{Local Contexts}

In a nutshell, we take the local context of an expression $E$ to be the minimal domain of objects that the interpreter needs to consider when he computes the contribution of $E$ to the meaning of the entire sentence. How can this notion of 'minimal domain' be motivated? The interpreter's task is to determine which worlds of the context set are compatible with the speaker's claim; in other words, he must compute a function from worlds in the context set to truth values. To do so, he has access to the context set $\mathrm{C}$, and to the meaning of the words, which we take for simplicity to be functions of various types ${ }^{2}$. Now we will assume (i) that it is easier to perform the steps of the computation when part of the domain of a function can be disregarded, (ii) that the interpretation is performed incrementally (from left to right), and (iii) that before processing any expression, the interpreter tries to simplify his task as much as possible given what he already knows about the meaning of the sentence. From these assumptions, it follows that the interpreter will try to determine in advance of interpreting any expression $E$ what is the smallest domain that he needs to consider when he assesses the meaning of $E$; this 'smallest domain' is our notion of local context.

Let us make the intuition clear with an example. Suppose that we are in a context $\mathrm{C}$, and that we have heard the speaker say: If John used to smoke, ... . Having computed the meaning of the antecedent of the conditional, we set out to compute the meaning of its consequent - call it $S$. For simplicity, we analyze the conditional as a material implication. Now one strategy would be to retrieve a function that specifies the value of $S$ in all possible worlds. But for the purposes of the conversation, only the worlds in $\mathrm{C}$ matter, because all other worlds are excluded by the shared assumptions of the conversation partners. So instead of computing the meaning of $S$, the interpreter may just as well retrieve a function which uniformly assigns the value 0 (for 'false) to all worlds outside of $\mathrm{C}$, and which assigns the value of $S$ to those worlds that lie in $\mathrm{C}$. With this procedure, the interpreter can assign 0's to all the worlds that are not within $\mathrm{C}$, and he can do so before computing the meaning of $S$. In effect, an interpreter that follows this strategy will compute the value of ( $c^{\prime}$ and $S$ ) instead of the value of $S$, where $c$ ' denotes C. For simplicity, we will describe this strategy by literally replacing $S$ with ( $c^{\prime}$ and $\left.S\right)$ t, though we are not committed to such a syntactic implementation of the procedure (semantic variants could be found, but they would be more cumbersome). We will say that the interpreter assesses $S$ with a restriction to $c^{\prime}$, and that this restriction is 'innocuous' because no matter what the value of $S$ turns out to be, the

\footnotetext{
${ }^{2}$ For perspicuity, we assimilate functions from individuals to truth values with sets of the individuals.
} 
restriction does not affect the truth conditions of the entire sentence relative to the context set. Still, the interpreter can make his life even easier by further restricting attention to those Cworlds in which John smoked, because all worlds in which John never smoked will make the conditional true no matter what the value of $S$ turns out to be; so if $c$ ' denotes the set of p-worlds within $\mathrm{C}$, c' will still be an innocuous restriction. We take the local context of $S$ to be the strongest innocuous restriction, i.e. the one that entails all other innocuous restrictions.

We will assume that the computation of the local context of an expression $E$ is done 'on the fly', as a sentence is processed from left to right. This gives rise to an asymmetry: information that comes before $E$ is known when the local context is computed, but information that comes after $E$ isn't, and the interpreter must therefore ensure that no matter how the sentence ends the local context will indeed be innocuous (this assumption is reconsidered below).

Before we go further, let us make sure that for a sentence $S$ uttered in a context $\mathrm{C}$, the local context is the global one, i.e. C itself. Certainly the worlds that don't lie in $\mathrm{C}$ are known to be irrelevant to the conversation, so the interpreter may compute the meaning of ( $c$ ' and $S$ ) rather than $S$, where $c$ ' denotes $C$. On the other hand, no further restriction will be innocuous. For suppose that $c$ ' denotes a set that excludes some world w of C. Since the interpreter doesn't yet know the value of $S$, it might turn out that $S$ is true in w; if so, by computing (c' and $S$ ) instead of $S$ the interpreter will reach the erroneous conclusion that the sentence is false in $\mathrm{w}$, since $c$ ' excludes w. So we have obtained two results: restricting attention to $\mathrm{C}$ is innocuous, and any innocuous restriction must include all of C. In other words, $\mathrm{C}$ is the strongest restriction that one can make before assessing $S$, and it is thus the local context of $S$. If $S$ is, say, the sentence John stopped smoking, we correctly predict that $\mathrm{C}$ should guarantee that John used to smoke (since the local context of $S$ must entail its presupposition).

Turning to a more sophisticated example, let us compute the local context of $S$ in the complex sentence John used to smoke and S. We ask once again what is the smallest domain of worlds that the interpreter may restrict attention to when he starts interpreting $S$. As before, he may exclude from consideration all worlds that are not compatible with $\mathrm{C}$. But now he can do more: any world $w$ in which John never smoked will make the first conjunct false, and thus the value in $\mathrm{w}$ of the second conjunct will be immaterial to the conversation. Hence it won't hurt to compute the meaning of $c^{\prime}$ and $S$ rather than $S$, where $c$ ' denotes those C-worlds that satisfy the first conjunct. On the other hand, all of these worlds must be considered. For suppose that $c^{\text {' }}$ excluded some world w of $\mathrm{C}$ in which John used to smoke, and suppose that $S$ is true in w; by computing John used to smoke and (c' and S) rather than John used to smoke and S, the interpreter would wrongly conclude that the sentence is false in w (since $c$ ' excludes $\mathrm{w}$ ). The set of C-worlds that satisfy the first conjunct is thus the strongest restriction that the interpreter can make without risk; in other words, it is the local context of the second conjunct. This correctly predicts that John used to smoke and he has stopped smoking does not presuppose anything: by construction, the local context of the second conjunct already entails its presupposition, and so no special demands are made on the global context $\mathrm{C}$.

\subsection{Formal Definitions}

To state our principle more precisely, we will make some simplifying assumptions. We work with a formal language that is structurally disambiguated by the use of parentheses. We will restrict attention to simple sentences of the form $p$, (not $F$ ), ( $F$ and $G$ ), ( $F$ or $G$ ), (if $F$. $G$ ), (No $P$ . R) and (Each P.R). The syntax of our fragment is defined in (5); it is standard, except that (a) 
quantifiers such as Each, some, none and most are treated as generalized quantifiers which take two predicative arguments (as is necessary when dealing with natural language quantification), and (b) elementary predicative or propositional presuppositions are underlined.

(5) Syntax

a. Predicates: $\mathrm{P}::=\mathrm{P}_{\mathrm{i}} \mid \underline{\mathrm{P}}_{\mathrm{i}} \mathrm{P}_{\mathrm{k}}$

b. Propositions: $p::=p_{i} \mid p_{i} p_{k}$

c. Formulas: $F::=p \mid($ not F $) \mid(F$ and F) $\mid(F$ or F $) \mid($ if F. F $) \mid($ Each P . P $) \mid($ No P . P ) |

(Most P . P)

By contrast with dynamic semantics, our interpretation function is bivalent and classical. It is standard, except that expressions of the form $\underline{P}_{i} P_{k}$ and $\underline{p}_{i} p_{k}$ are interpreted as the (predicative or propositional) conjunction of their two components. For notational simplicity, we write as $\mathbf{E}$ the semantic value of an expression $E$; and we write as $\mathbf{E}^{\mathrm{w}}$ the value of $E$ evaluated in a world w.

(6) Semantics

We take as given a domain $\mathrm{D}$ of individuals and a domain $\mathrm{W}$ of possible worlds.

The initial valuation assigns to each elementary predicate $P_{i}$ a value $\mathbf{P}_{\mathbf{i}}{ }^{\mathrm{w}} \subseteq \mathrm{W}$ and to each elementary proposition $p_{i}$ a value $\mathbf{p}_{\mathbf{i}}{ }^{\mathrm{w}} \in\{0,1\}$. For any world $\mathrm{w}$ of $\mathrm{W}$ :

a. $\left(\mathbf{p}_{i} \mathbf{p}_{\mathbf{k}}\right)^{\mathrm{w}}=1$ iff $\mathbf{p}_{\mathbf{i}}{ }^{\mathrm{w}}=\mathbf{p}_{\mathbf{k}}{ }^{\mathrm{w}}=1 ;\left(\underline{\mathbf{P}}_{\mathrm{i}} \mathbf{P}_{\mathbf{k}}\right)^{\mathrm{w}}=\mathbf{P}_{\mathbf{i}}^{\mathrm{w}} \cap \mathbf{P}_{\mathbf{k}}{ }^{\mathrm{w}} ;$

b. $(\boldsymbol{n o t} \mathbf{F})^{\mathrm{w}}=1$ iff $\mathbf{F}^{\mathrm{w}}=0$; $\left(\mathbf{F} \text { and } \mathbf{F}^{\prime}\right)^{\mathrm{w}}=1$ iff $\mathbf{F}^{\mathrm{w}}=\mathbf{F}^{\text {, }}{ }^{\mathrm{w}}=1 ;\left(\mathbf{F} \text { or } \mathbf{F}^{\prime}\right)^{\mathrm{w}}=1$ iff $\mathbf{F}^{\mathrm{w}}=1$ or $\mathbf{F}^{\text {, }}$

$=1$; $\left(\text { if } \mathbf{F} . \mathbf{F}^{\prime}\right)^{\mathrm{w}}=1$ iff $\mathbf{F}^{\mathrm{w}}=0$ or $\mathbf{F}^{\boldsymbol{y}_{\mathrm{w}}}=1$; $\left(\text { Each } \mathbf{P} . \mathbf{P}^{\prime}\right)^{\mathrm{w}}=1$ iff Each object $\mathrm{d} \in \mathrm{D}$ such that

$\mathrm{d} \in \mathbf{P}^{\mathrm{w}}$ satisfies $\mathrm{d} \in \mathbf{P}^{{ }^{\mathrm{w}}} ;\left(\mathbf{N o} \mathbf{P} . \mathbf{P}^{\prime}\right)^{\mathrm{w}}=1$ iff no object $\mathrm{d} \in \mathrm{D}$ such that $\mathrm{d} \in \mathbf{P}^{\mathrm{w}}$ satisfies $\mathrm{d} \in$

$\left.\mathbf{P}^{\mathrm{w}} ; \mathbf{P}^{\text {Most } \mathbf{P}} . \mathbf{P}^{\prime}\right)^{\mathrm{w}}=1$ iff more than half of the objects $\mathrm{d} \in \mathrm{D}$ such that $\mathrm{d} \in \mathbf{P}^{\mathrm{w}}$ satisfy $\mathrm{d} \in$ $\mathbf{P}^{\prime}$

Thus the semantics treats the presuppositional component $d$ of an elementary expression $\underline{d} d$ ' as if $d$ were just part of its assertive component. But the pragmatics will give $d$ a distinguished status through the requirement that the presupposition of an elementary expression be entailed by its local context.

How are local contexts computed? Since presupposition triggers are either of predicative or propositional type, we only need to determine the local context of propositional and predicative expressions. To do so, we enrich the object language with (a) context variables, which may be of propositional or predicative type (they will be written below as $c^{\prime}$ ), and (ii) predicate conjunction, which receives the natural interpretation. If $\mathrm{w}$ is a world and $F$ is a formula (which may contain the variable $c^{\prime}$ ), we write $\mathrm{w} \mid={ }^{\mathrm{c}^{\prime} \rightarrow \mathrm{x}} \mathrm{F}$ to indicate that $\mathrm{w}$ satisfies the formula $F$ when $c^{\prime}$ denotes $\mathrm{x}$; if $\mathrm{C}$ is a set of worlds, $\mathrm{C} \mid=^{\mathrm{c}^{\prime} \rightarrow}{ }^{\mathrm{x}} \mathrm{F}$ indicates that each world in $\mathrm{C}$ satisfies $F$ when $c^{\prime}$ denotes $\mathrm{x}$. We say that a proposition $\mathrm{x}$ is a stronger than a proposition $\mathrm{x}^{\prime}$ if $\mathrm{x}$ entails $x^{\prime}$; and similarly if $x$ and $x$ ' are properties, with a generalized notion of entailment ${ }^{3}$. Our definition of local contexts can now be stated as follows:

(7) The local context of an expression $d$ of propositional or predicative type which occurs in a syntactic environment $a_{-} b$ in a context $\mathrm{C}$ is the strongest proposition or property $\mathrm{x}$ which

\footnotetext{
${ }^{3}$ Writing as $\mathrm{x}^{\mathrm{w}}$ the value of a property $\mathrm{x}$ at $\mathrm{w}, \mathrm{x}$ entails a property $\mathrm{x}$ ' just in case for every possible world $\mathrm{w}$ and for every individual $d$, if $d \in x^{w}$, then $d \in x^{\prime}$.
} 
guarantees that for any expression $d^{\prime}$ 'of the same type as $d$, for all strings $b^{\prime}$ for which $a$ $d^{\prime} b$ ' is a well-formed sentence,

$\mathrm{Cl}=\mathrm{I}^{\mathrm{c}^{\prime} \rightarrow \mathrm{x}}$ a (c' and d') b' $\Leftrightarrow \mathrm{a} \mathrm{d}^{\prime} \mathrm{b}^{\prime}$

(If no strongest proposition or property $\mathrm{x}$ with the desired characteristics exists, the local context of $d$ does not exist $\left.{ }^{4}\right)$.

Following the intuition we developed earlier, the local context of $d$ in the sentence $a d b$ is the strongest innocuous restriction that the interpreter can make in advance of interpreting $d$ when he processes the sentence from left to right. At this point, he has access to the meaning of the expressions in the string $a$, but not to the meaning of $d$ itself, nor to the string $b$. Our procedure is incremental because it requires that the local context be an innocuous restriction no matter which expressions appear at the end of the sentence. This is why the rule in (7) involves a quantification not over over $d^{\prime}$ ' (since the value of $d$ is not known yet), but also over $b^{\prime}$ (since the end of the sentence has not been heard yet): for every appropriate $d^{\prime}$ and $b^{\prime}, a$ (c' and $\left.d^{\prime}\right) b^{\prime}$ should be equivalent to $a d^{\prime} b$ ' relative to the context set.

With this notation in place, we can repeat the standard definition of presupposition satisfaction offered within the dynamic tradition:

(8) An elementary presuppositional expression $E$ is acceptable in a sentence $S$ uttered in a context $\mathrm{C}$ just in case the presupposition of $E$ is entailed by the local context of $E$ (if it exists).

\subsection{Examples}

Let us see how this analysis derives some standard results of dynamic semantics. In the metalanguage, we write as $\wedge$ the conjunction of propositions or properties. In Section 2.1., we informally derived the results in (9):

(9) a. pp': the local context of pp' is $\mathrm{C}$, which must thus entail $\mathrm{p}$.

b. ( $\left(\mathrm{p}\right.$ and $\left.q q^{\prime}\right)$ : the local context of qq' is $\mathrm{C} \wedge \mathrm{p}$, which must thus entail $q$.

We already sketched the beginning of the reasoning for conditionals:

(10) (if $\mathrm{p}$. qq'): the local context of $q q^{\prime}$ is $\mathrm{C} \wedge \mathbf{p}$, which must thus entail $q$

As was noted, when we assess the consequent after processing the meaning of the antecedent, it is innocuous to restrict attention to those $\mathrm{C}$-worlds that satisfy $p$ : if $c$ ' denotes $\mathrm{C} \wedge \mathbf{p}$, we can be certain that throughout $\mathrm{C}$ (if $p$. ( $c^{\prime}$ and $S$ )) has the same value as (if $p$. $S$ ). On the other hand, any world $\mathrm{w}$ in $\mathrm{C} \wedge \mathbf{p}$ could turn out to matter. For suppose that $p$ is true $\mathrm{w}$, and that $c$ ' excludes $\mathrm{w}$. If $S$ is true in w, by computing (if $p$. (c' and $S$ )) instead of (if $p . S$ ), we will reach the erroneous conclusion that the entire conditional is false in $w$. So any innocuous restriction must include all of $\mathrm{C} \wedge \mathbf{p}$, which is thus the local context of $q q^{\prime}-$ and it must entail the presupposition $q$.

\footnotetext{
${ }^{4}$ See Schlenker (to appear) for a discussion of the case in which local contexts do not exist.
} 
Turning to the case of disjunctions, we saw in (3)b that in a sentence ( $p$ or $q q^{\prime}$ ) the presupposition of the second disjunct can be justified by the negation of the first disjunct. This fact is encoded in the dynamic meaning of or posited by some theorists (e.g. Beaver 2001), but dynamic semantics does not derive it from first principles. We do: using our general algorithm, we can see that the local context of the second disjunct is $\mathrm{C} \wedge(\operatorname{not} \mathbf{p})$ :

(11) (p or qq'): the local context of $q q^{\prime}$ is $\mathrm{C} \wedge($ not $\mathbf{p})$, which must thus entail $q$

First, we note that for the familiar reason a restriction to $\mathrm{C}$-worlds innocuous. But we can do more: by propositional logic, $(p$ or $S$ ) is equivalent to ( $p$ or ((not $p)$ and $S)$ ), which shows that a restriction to (not p)-worlds is innocuous as well. To show that this is the strongest innocuous restriction, let us assume that we compute ( $p$ or ( $c$ ' and $S$ )) instead of $S$, where $c$ ' excludes some world $\mathrm{w}$ that lies in $\mathrm{C} \wedge(\boldsymbol{n} \operatorname{not} \mathbf{p})$. If $S$ is true in $\mathrm{w}$, we will get the erroneous impression that the disjunction is false in $\mathrm{w}$, since $p$ and $c$ ' are both false in $\mathrm{w}-$ whereas ( $p$ or $S$ ) is true in w. So the local context of the second disjunct is $\mathrm{C} \wedge(\operatorname{not} \mathbf{p})$, which must thus entail the presupposition $q$.

We also make correct predictions for some quantified examples. As noted, Each of my students has stopped smoking and None of my students have stopped smoking both presuppose that each of my students used to smoke. The universal inference obtained in the case of none is particularly important because it is characteristic of presuppositions - neither entailments nor scalar implicatures give rise to such a pattern (see Chemla, to appear, for discussion and experimental data from French). To derive this observation, we must first ask what is the local context of the verb phrase $V$ in structures of the form (Each $P$. $V$ ) or (No $P$. $V$ ). Since $V$ is of predicative type, its local context must be predicative as well; by definition, it is the strongest innocuous restriction that one can add to $V$. If we write ${ }^{\mathrm{C}} \mathbf{P}$ for the property $\mathbf{P}$ restricted to $\mathrm{C}$, i.e. the property whose extension is empty at any world which is not in $\mathrm{C}$, and which is co-extensive with $\mathbf{P}$ at worlds that are in $\mathrm{C}$, we obtain the result in (12) (with $\mathrm{V}=\underline{Q} \mathrm{Q}^{\prime}$ ):

(12) (No P . QQ'), (Each P . QQ'): the local context of $Q Q$ ' is ${ }^{\mathrm{C}} \mathbf{P}$, which must thus entail $Q$

As before, the reasoning is in two steps. We illustrate it in the case of (No P. QQ'). First, we must show that the restriction to ${ }^{\mathrm{C}} \mathbf{P}$ is innocuous, in the sense that when $c$ ' denotes ${ }^{\mathrm{C}} \mathbf{P}$ one may without risk compute (No $P$. ( $c^{\prime}$ and $\left.V\right)$ ) rather than (No $P$. V). The exclusion of non-C worlds is unproblematic; and because of the meaning of no, (No $P . V$ ) is always equivalent to (No $P$. ( $P$ and $V)$ ), which means that one can safely restrict attention to P-individuals. Second, we must show that any further restriction would carry a risk. So suppose that there is a pair $<\mathrm{w}, \mathrm{x}>$ such that $\mathrm{x}$ has property ${ }^{\mathrm{C}} \mathbf{P}$ in $\mathrm{w}$, and that $c$ ' is false of $<\mathrm{w}, \mathrm{x}>$. If $V$ is true of $<\mathrm{w}, \mathrm{x}>$ and false of all other pairs, by computing (No P. (c' and V)) instead of (No $P$. $V$ ) we will get the erroneous impression that the sentence is true in $\mathrm{w}$, whereas it is in fact false (because of $\mathrm{x}$, which satisfies $P$ and $V$ in w). So we cannot exclude $<\mathrm{w}, \mathrm{x}>$ from consideration ${ }^{5}$. The conclusion is that ${ }^{\mathrm{C}} \mathbf{P}$ is the local context of the verb phrase, and that it must thus entail its presupposition $Q$.

\footnotetext{
${ }^{5}$ The reasoning is similar for (Each $\left.P . Q Q^{\prime}\right)$. When $c^{\prime}$ denotes ${ }^{\mathrm{C}} \mathbf{P},($ Each $P$. ( $c$ ' and $\left.V)\right)$ is equivalent to $($ Each $P$. V): the restriction to ${ }^{\mathrm{C}} \mathbf{P}$ is innocuous. To show that this is the strongest innocuous restriction, let us assume that there is a pair $<\mathrm{w}, \mathrm{x}>$ for which $\mathrm{x}$ has property ${ }^{\mathrm{C}} \mathbf{P}$ in $\mathrm{w}$, and that $c^{\prime}$ is false of $<\mathrm{w}, \mathrm{x}>$. If $V$ is a tautological predicate, by computing (Each P. ( $c^{\prime}$ and $\left.V\right)$ ) instead of (Each P. V) we will get the erroneous impression that the sentence is false in $\mathrm{w}$ (because in $\mathrm{w} x$ satisfies $P$ but not $c^{\prime}$ ) - although it is in fact true. So the restriction to $\mathrm{x}$ is not innocuous. This shows that ${ }^{\mathrm{C}} \mathbf{P}$ is the local context of $Q Q$ '.
} 


\subsection{General Results}

In the general case, it can be shown that under relatively mild assumptions, (i) local contexts are guaranteed to exist, and that (ii) the present theory makes almost the same predictions as Heim's dynamic semantics for a fragment similar to the one she considered - supplemented with the disjunction of Beaver 2001 (see also Schlenker, 2007, to appear; some experimental results involving other quantifiers are problematic both for Heim's theory and for the present analysis, as is discussed in Chemla, to appear).

\section{Extensions}

The present account lends itself to two extensions. Unlike dynamic semantics, it offers a natural account of 'symmetric' readings, in which a presupposition is justified by virtue of linguistic elements that come later in the sentence. And unlike non-dynamic analyses, it yields a general account of the contribution of an expression to the truth conditions of a sentence - which paves the way for a general theory of triviality.

\subsection{Symmetric Readings}

At this point our account follows standard dynamic semantics in predicting, incorrectly, that there should be a clear contrast between the following sentences:

(13) a. There is no bathroom or the bathroom is well hidden (after Partee).

b. The bathroom is well hidden or there is a no bathroom.

a'. If there is a bathroom, the bathroom is well hidden.

$\mathrm{b}^{\prime}$. If the bathroom is not hidden, there is not bathroom.

(13)a-a' are correctly predicted to carry no presupposition. By contrast, theories with a left-right bias (be it dynamic semantics or the present analysis) predict that (13)b-b' should presuppose that there is a bathroom ${ }^{6}$. The issue is complex and would require a longer discussion (see Schlenker 2008a, and Geurts 1999). It is plausible that in these examples the presupposition of the first element is justified on the basis of information that appears at the end of the sentence; in fact, when the entire sentence is taken into account, (13)b becomes informationally indistinguishable from (13)a. And similarly for (13)b' and (13)a': trading on the nearequivalence between If not $F$, not $G$ and if $G, F$, when the entire sentence is taken into account, (13)b' becomes informationally similar to (13)a' - which makes it unsurprising that they should transmit presuppositions in the same way. We take these observations to suggest that the local context of an expression $E$ in a sentence $S$ can to some extent be computed by taking into account all of $S$ except $E$. This option is presumably costly, since (13)b-b' are somewhat less felicitous than (13)a-a'. Still, it appears that the left-right asymmetry we observed is just a bias, which can be overcome with some effort, rather than a rigid property of the system, as is

\footnotetext{
${ }^{6}$ Post-posed if-clauses also make the same point: The bathroom is well-hidden, if there is a bathroom is much more acceptable than is predicted by the incremental version of our analysis; on the other hand, the symmetric version makes appropriate predictions (for this particular example, Heim's dynamic semantics makes correct predictions, which differ from those of the incremental version of our theory; by contrast, in (13)b' the basic version of Heim's theory and our incremental theory both make incorrect predictions).
} 
postulated by standard dynamic semantics (see Chemla and Schlenker 2009 for experimental evidence that symmetric readings are generally possible, albeit somewhat degraded; the symmetric version of the analysis is developed in greater detail in Schlenker, to appear) ${ }^{7}$.

\subsection{Triviality}

Like dynamic semantics, our theory offers an immediate account of the contribution of an expression to its local context, which was precisely what Stalnaker was trying to achieve. We may call 'local meaning' of $E$ the intersection of $\mathbf{E}$ with the local context of $E$. An expression $E$ is locally trivial if the local meaning of $E$ is indistinguishable from that of a tautology or of a contradiction - which is to say that $E$ or (not $E$ ) is entailed by its local context (see Stalnaker 1978). This immediately accounts for the pragmatic oddness of the examples in (14): in each case, the expression in bold is either entailed by its local context, or contradictory with it.

(14)a. ? John has cancer and he is sick.

b. ? John is sick or he has cancer.

c. ? No student is a student and proud of it.

Given his analysis of conjunctions, Stalnaker could account for the oddness of (14)a, since he is sick is entailed by its local context. But for lack of a general theory of local contexts, (14)b-c weren't explained. They are in the present framework: in (14)b, he has cancer is contradictory in its local context, which entails the negation of John is sick. In (14)c, a student is entailed by its local context. This analysis could in principle pave the way for a more general theory of triviality.

\section{Conclusion}

We take the present exercise to show that a theory very much in the spirit of Stalnaker's pragmatic analysis can be made both general and formally precise. Importantly, this means that presuppositions do not provide an argument for postulating a dynamic semantics: a (modified) dynamic pragmatics is enough. But this result can be achieved only if one grants that a local

\footnotetext{
${ }^{7}$ The simplest way to compute the local context of an expression $d$ that occurs in a syntactic environment $a \_b$ is to find the strongest $\mathrm{x}$ which guarantees that for any expression $d$ ' of the same type as $d$,

(i) $\mathrm{C} \mid=^{\mathrm{c}^{\prime} \rightarrow \mathrm{x}}$ a (c' and d') $\mathrm{b} \Leftrightarrow \mathrm{a} \mathrm{d}^{\prime} \mathrm{b}$

(i) is similar to the rule in (7), except that the string $b$ is taken as given (by contrast, in (7) we required that the equivalence hold no matter which string b' comes at the end of the formula). But within our bivalent semantics, such an implementation leads to incorrect predictions. For instance, in ( $p p^{\prime}$ and $q q$ '), we predict that the presupposition of each conjunct could be satisfied by the presupposition of the other - with the result that for $q q^{\prime}=p p^{\prime}$, the sentence should presuppose nothing at all (see Beaver 2008, Rothschild 2008, and 2008b, to appear for discussion). This problem can be circumvented by defining for every string $s$ a string $s^{*}$ obtained by deleting all underlined material from $s$, and replacing (i) above with (ii), which has the effect of requiring that a local context be computed only on the basis of the assertive component of expressions that are found in a sentence:
}

(i) $\mathrm{C} \mid=^{\mathrm{c}^{\prime} \rightarrow \mathrm{x}} \quad \mathrm{a}^{*}\left(\mathrm{c}^{\prime}\right.$ and $\left.d^{\prime}\right) \mathrm{b}^{*} \Leftrightarrow \mathrm{a}^{*} \mathrm{~d}^{\prime} \mathrm{b}^{*}$ 
context is a shortcut to facilitate sentence interpretation, rather than the result of some process of belief update.

\section{References}

Beaver, David. 2001. Presupposition and Assertion in Dynamic Semantics. CSLI, Stanford.

Chemla, Emmanuel and Schlenker, Philippe. 2009. Incremental vs. Symmetric Accounts of Presupposition Projection: An Experimental Approach. Manuscript, Institut Jean-Nicod and LSCP.

Chemla, Emmanuel. To appear. Presuppositions of Quantified Sentences: Experimental Data. To appear in Natural Language Semantics.

Heim, Irene. 1983. On the Projection Problem for Presuppositions. In D. Flickinger et al. (eds), Proceedings of the Second West Coast Conference on Formal Linguistics, 114-125. Reprinted in Davis 1991.

Heim, Irene. 1990. Presupposition Projection. In R. van der Sandt (ed.), Reader for the Nijmegen Workshop on Presupposition, Lexical Meaning, and Discourse Processes. U. of Nijmegen.

Heim, Irene. 1992. Presupposition projection and the semantics of attitude verbs. Journal of Semantics, 9(3), 183-221.

Karttunen, Lauri. 1974. Presupposition and Linguistic Context. Theoretical Linguistics 1: 181194. Reprinted in Davis 1991.

Schlenker, Philippe. 2007. Anti-Dynamics: Presupposition Projection Without Dynamic Semantics. Journal of Logic, Language and Information 16, 3: 325-256.

Schlenker, Philippe. 2008a. Be Articulate: A Pragmatic Theory of Presupposition Projection". Theoretical Linguistics, 34, 3: 157-212

Schlenker, Philippe. 2008b. Presupposition Projection: Explanatory Strategies. Theoretical Linguistics 34, 3: 287-316

Theoretical Linguistics

Schlenker, Philippe. To appear. Local Contexts. To appear in Semantics and Pragmatics.

Soames, Scott. 1989. Presupposition. In D. Gabbay and F. Guenthner (eds), Handbook of Philosophical Logic IV, 553-616.

Stalnaker, Robert. 1974. Pragmatic Presuppositions. In Munitz, M. and Unger, P. (eds.) Semantics and Philosophy. New York: New York University Press. Reprinted in Davis 1991.

Stalnaker, Robert. 1978. Assertion. Syntax and Semantics 9, Academic Press, New York. 